\title{
Communio et Progressio: „Entgegenstehendes ist hiermit außer Kraft gesetzt."
}

\author{
von Ambros Eichenberger
}

Im Hinblick auf die rasanten und teilweise unübersichtlichen Entwicklungen, vor allem im Bereich der sog. neuen Medien, kann es geradezu einen anachronistischen Eindruck machen, wenn man sich zur "Feier“ eines Mediendokumentes zusammenfindet, das bereits vor 20 Jahren entstanden ist. ${ }^{1}$ Technologiesprünge im Bereich der Mikroelektronik, ständig ansteigende Datenübertragungsgeschwindigkeiten, das relativ neue duale System von öffentlich-rechtlichen und privaten Anbietern usw. haben neue Szenarien und Paradigmen geschaffen, deren Auswirkungen auf Kultur und Kreativität, aber auch auf den Alltag, die Arbeitswelt und die Freizeitgestaltung der Menschen noch gar nicht abzusehen sind. Man kann dementsprechend belegen, daß das, was am Anfang der 80er Jahre über die technischen und gesellschaftlichen Auswirkungen neuer Medien veröffentlicht wurde, in den meisten Fällen heute kaum noch das Papier wert ist, auf dem es geschrieben wurde.

Mit dem Fortschritt dieser Medientechnologien, die einerseits das Wahrnehmungs- und Erlebnispotential beträchtlich erweitert haben, ist andererseits auch die Kehrseite der Medaille, die Ambivalenz der Medien, stärker ins Bewußtsein gerückt. So kennen wir z. B. die nicht ganz unbegründete Angst vor dem totalen elektronischen Überwachungsstaat. Der Golfkrieg hat deutlich gemacht, bis zu welchem Grade die kommunikationstechnologischen Entwicklungen militärischen Zielen dienstbar gemacht werden können. Und das Anwachsen von global und multimedial agierenden Megakonzernen mit neuen Marktund Industriestrukturen, welche die publizistisch-kulturelle Funktion der Medien zunehmend in Frage stellen, haben den wirtschaftlichen Faktor im Medienwettbewerb ins Zentrum gerückt, was nicht heißt, daß die Anteils- und Besitzstrukturen durchschaubarer geworden sind. Säkulare Propheten wie z. B. Neil Postman oder Herbert Schiller haben deshalb, nicht ohne Grund, vor der zunehmenden "Industrialisierung des Bewußtseins" und der "geistigen Entsorgung" durch den sich ausbreitenden Trend zum sog. "Infotainment" gewarnt. „Ein Sieg des Business über Kunst“ -, mit diesen Worten hat ein Beobachter bei den diesjährigen Berliner Filmfestspielen seine Eindrücke zusammengefaßt.

Die eingetretene Ernüchterung hat zur Folge, daß Leitbilder, wie sie mit den Begriffen "Communio" (Gemeinschaft der Menschen) und "Progressio" (Fortschritt der menschlichen Gesellschaft) mehr angeritzt als entfaltet wurden, heute nicht mehr so unkritisch übernommen werden können wie vor 20 Jahren.

Pater Ambros Eichenberger war von 1983 bis 1990 OCIC-Präsident und arbeitet gegenwärtig als Filmreferent beim Katholischen Mediendienst der Schweizer Bischofskonferenz in Zürich. 
So wird z. B. der Begriff „Fortschritt“ neu zu definieren sein, z: B. durch ein vernetztes, interdisziplinäres Denken, das humane, soziale, ökologische und philosophische Aspekte mit den kommunikationstechnologischen viel stärker als bisher in Verbindung bringt. Auch die Aussage, daß die Kirche in den Medien "Geschenke Gottes" (CP 1) erblickt, „weil sie nach dem Ratschluß der göttlichen Vorsehung die Menschen brüderlich verbinden“ (CP 2) findet heute kaum mehr ungeteilte Zustimmung, auch wenn sie der Papst in der Botschaft zum diesjährigen Mediensonntag nach wie vor verwendet.

Das haben bekanntlich nicht alle seine Vorgänger getan! Vor dem „Epochensprung", den die katholische Kirche (im Prinzip) mit dem 2. Vatikanischen Konzil vollzogen hat, beherrschten weitgehend Abwehr, Mißtrauen, Distanz und Zensur die kirchliche Einstellung gegenüber den Medien und den Medienschaffenden. „Le Cinéma est une école où les garçons apprennent à voler et à faire le coup de poing", gab ein römischer Prälat im Jahre 1927 zu Protokoll. Die Aussage illustriert den negativen Ansatz recht anschaulich und gut! Neun Jahre später hat Papst Pius XI. mit seiner Filmenzyklika, die den bezeichnenden Titel „Vigilanti Cura“ trägt, dann - allerdings um einiges differenzierter - nachgedoppelt. All diese Texte, sowohl die positiven wie die negativen, müssen vom zeitlichen Hintergrund her gelesen werden, in dem sie entstanden sind. „Communio et Progressio" ist ein Kind des 2. Vatikanischen Konzils, das ja auch den Auftrag zur Abfassung dieses Schreibens gegeben hat, weil das vorausgehende Medien-Dokument „Inter Mirifica“ die Mehrzahl der Konzilsväter nicht zu befriedigen vermochte.

Sieben Jahre intensiver Arbeit mit weltweiten Konsultationen sind der Publikation des Textes am 3. Juni 1971 vorausgegangen. Die Verfasser sollen dabei bestrebt gewesen sein, den offenen Kommunikationsstil mit den demokratischen Grundwerten der freien Meinungsäußerung, des Rechtes auf Information, der Toleranz anderen Auffassungen gegenüber, den Forderungen nach Transparenz, die sie in der Instruktion schriftlich festgehalten haben, selbst in die Praxis umzusetzen. „Der Arbeitsprozeß hat den Versuch dargestellt, in der Kirche einen echten Dialog zu erreichen“, bemerkte ein Mitglied der damaligen päpstlichen Medienkommission in diesem Zusammenhang.

Der grundsätzliche Teil des Textes hat seine Bedeutung denn auch bis heute nicht verloren und er soll sie nicht verlieren, wenn man der prägnanten kirchenrechtlich bindenden Schlußformel Glauben schenkt: „Entgegenstehendes ist hiermit außer Kraft gesetzt." Es ist allerdings viel leichter gesagt als getan. Darüber haben sich schon damals verschiedene Kommentatoren keine Illusionen gemacht. Eine italienische Zeitung zitiert z. B. den römischen Korrespondenten von „Le Monde“, der die Aufassung vertrat, „daß die Instruktion ohne eine langwierige Sinnesänderung und ohne eine wirksame Reform der kirchlichen Informationsstrukturen, die noch immer vorwiegend von oben nach unten operieren, nicht werde angewendet werden können."

Und der bekannte italienische Regisseur Ermanno Olmi gab zu bedenken, daß es sich zwar um ein ausgezeichnetes Dokument handle, „das $40-$ 
50 Jahre zu spät komme" und „daß in einem Augenblick wie dem jetzigen Worte allein nicht mehr genügen, jenen Pessimismus zu überwinden, der von Zeit zu Zeit aufbreche“.

Das war vor 20 Jahren! Aber die Vermutung liegt nahe, daß viele noch heute oder heute erst recht ähnlich empfinden. Stellvertretend sei Michael Schmolke zitiert, der vor kurzem in der Zeitschrift "Communicatio Socialis" geschrieben hat: „Ein besseres Kommunikationsgrundgesetz als CP ist schwer denkbar - wenn es nur endlich überall beachtet und verwirklicht würde." Damit ist auf ein recht beträchtliches Vollzugs- und Rezeptionsdefizit hingewiesen, das vor allem auch innerhalb der Kirche selbst festzustellen ist. Die aufgestellten Forderungen sind ja nicht in erster Linie an andere gerichtet. Um glaubwürdig zu sein, sollten sie zuerst in den eigenen Reihen verwirklicht werden. Deshalb wurde ja auch der Vorschlag gemacht, im Zusammenhang mit dem Jubiläum „eine gründliche und weltweite Untersuchung darüber anzustellen, in welchem Maße die Aussagen der Instruktion rezipiert und ihre Forderungen und kommunikationstheoretischen Prinzipien in den vergangenen 20 Jahren realisiert worden sind.“ (W. Schätzler, Sekretär der deutschen Bischofskonferenz)

\section{II.}

Eine solche Gewissensforschung und Standortbestimmung wird sich auch sehr offen mit den aktuellen Kommunikationsströmungen in der Kirche zu befassen haben, wenn sie ernst genommen werden soll. An Beispielen von Methoden, die mit den Grundsätzen von $\mathrm{CP}$ nicht zu vereinbaren sind, fehlt es wahrhaftig nicht. Man braucht sie nicht einmal in Lateinamerika, z. B. beim Redeverbot von Befreiungstheologen, zu suchen. Wenn fundamentalistische Kreise hierzulande, auch bei sorgfältig recherchierter Aufdeckung von Ungereimtheiten durch Journalisten (ich weiß, daß es auch andere gibt), bereits den Refrain von einem "gigantischen Medienmißbrauch“ mit der "totalen Irreführung des Publikums" anstimmen, dann ist das ein massiver Rückfall in reaktionäres, vorkonziliäres publizisitisches Verhalten. Denn man kann ja nicht so tun, als ob der Fall Kopp, der Fall Barschel, der Fall Marcinkus, der Fall Haas usw. von den Medien erfunden worden wäre. Solche Anschuldigungen erinnern an die alten griechischen Könige, die jeweils den Boten getötet haben, wenn er ihnen eine schlechte Botschaft brachte. Sie offenbaren eine defensive Bunkermentalität, wonach die Kirche der dämonischen Macht der Medien entgegenzuwirken habe. Es sei denn, diese bösen Medien lassen sich instrumentalisieren und zur Darstellung der eigenen Anliegen gebrauchen.

Dieser einseitige Instrumentalisierungstrend, der sich mit Hilfe von finanzstarken Gruppen auf agressive Weise auszubreiten scheint, beinhaltet eine enge Vorstellung von Pastoral, Evangelisation und Reevangelisation. Von der befreienden Vision, daß „Kommunikation um der Kommunikation willen“ auch einen "christlichen" Wert darstellt, wie das CP nachdrücklich unterstreicht, ist dabei nicht mehr viel zu spüren. Selbst elektronische Großeinsätze in den USA, bei denen die Religion und das Evangelium für spektakuläres Showbusiness mißbraucht worden sind, haben keine abschreckende Wirkung hinterlassen. 
Widersprüche zu den Grundsätzen der Instruktion von 1971 sind auch in einer Instruktion von 1990 über „Die kirchliche Berufung der Theologen“ auszumachen. Denn danach sind Meinungsverschiedenheiten von Theologengruppen - oder Laien - mit dem kirchlichen Lehramt, z. B. über Sexualmoral, kirchenpolitische Maßnahmen usw., kein Fall für die Öffentlichkeit. Öffentlichkeit in Sachen des Glaubens und der Sitten darf nur dort sein, wo sie durch die lehramtlichen Aussagen selbst hergestellt wird. Solche Aussagen lassen sich schwerlich mit dem vereinbaren, was CP unter Ziffer 25 über die Notwendigkeit und über das Zustandekommen der öffentlichen Meinung sagt. Sie erinnern an frühere Zeiten, wo man die Zwangsmaßnahmen, z. B. mit der Verweigerung der kirchlichen Druckerlaubnis, eine angebliche oder reale Verunsicherung der Gläubigen zu verhindern suchte. Das ist in unseren pluralistischen offenen Informationsgesellschaften, die auf den öffentlichen Diskurs und auf die freie Meinungsäußerung aller angewiesen sind, nicht mehr möglich. Nur wenn der Medienrezipient gut informiert wird, und er sich selbst gut informiert, „kann er einen aktiven und bedeutsamen Beitrag in die Gesellschaft einbringen", heißt es unter Ziffer 34 in Communio et Progressio. Von dieser Pflicht kann heute auch das kirchliche Leben und das kirchliche Lehramt niemanden dispensieren!

\section{III.}

Die Folgerungen, die aus den hier angedeuteten Veränderungen im Kommunikationsbereich von Kirche und Öffentlichkeit gezogen werden, lauten unterschiedlich. Die einen äußern unverholen: Wir sind um unsere Hoffnungen betrogen worden und verfallen dem Kulturpessimismus und der Resignation. Andere gehen scheinbar gelassen über den Relevanzschwund des kirchlich christlichen Kommunikationsangebotes hinweg, indem sie sich in eine Zeitphase zurückversetzen, wo Fürstbischöfe und Landesherren ihnen einen bequemen Majoritässtatus garantierten.

Als dritter Weg wäre eine aggressive Variante ins Auge zu fassen mit der Absicht, dem Geburtstagskind CP auf der Basis von Sachkenntnis und Durchhaltewillen mit einer Effizienzspritze zu neuem Leben zu verhelfen. Dafür sind die Grundoptionen und Grundsätze des Dokuments im Hinblick auf Gegenwart und Zukunft zu prüfen und weiterzuentwickeln. Die Einladung dazu ist im Text der Instruktion ja bereits enthalten, denn die Autoren haben ihn nicht als Abschluß, sondern als Anfang eines breit angelegten Reflexions- und Handlungsprozesses verstanden, der weitergehen soll. (CP 186)

Das sog. „Supplementum“, das vom päpstlichen Medienrat in absehbarer Zukunft veröffentlicht werden soll, vermag diese Leistung allerdings kaum zu erbringen. Schon deshalb nicht, weil es zu viele Repetitionen von Sachverhalten enthält, die vor 20 Jahren besser und prägnanter artikuliert worden sind. Dazu geschieht es vielfach mit den üblichen moralisierenden Appellen, die eher Ermüdung verursachen als Begeisterung! Neu, und deshalb beachtenswert, ist das Plädoyer für die Entwicklung von Medienstrategien, um die kirchliche Medienarbeit national und international auf effektivere Füße zu stellen: Zum Beispiel durch eine verläßlichere Mitwirkung der Einzelkirchen, auch hinsichtlich einer finanziellen Minimalausstattung, speziell in Bezug auf die internationalen katho- 
lischen Medienorganisationen, die heute im Zeitalter der Konzentrationen einen neuen, größeren Stellenwert bekommen sollten.

Erfreulich ist, daß gleich zu Beginn dieses „Appendix“ - der den feierlichen und hochgestelzten Titel „Novae Aetatis Initium“ trägt - bekräftigt wird, daß die Prinzipien und Erkenntnisse von CP ihre volle Gültigkeit behalten (vgl. Nr. 1, 2. Absatz). Bei der Vertiefung dieser Erkenntnisse wird man sich aber zu fragen haben, worin denn der spezifische Beitrag der Kirchen zu der heutigen Medienlandschaft mit ihren neuen Herausforderungen überhaupt noch bestehen könne. Das medientechnische, das medienwissenschaftliche und erst recht das medienwirtschaftliche Know How beherrschen andere Kräfte der Gesellschaft nämlich besser. Auch darüber haben sich die Autoren von $\mathrm{CP}$ keine Illusionen gemacht. Mit dem Satz, „daß ihr, d. h. der Kirche, wertvollster und vorrangigster Beitrag nur auf geistigem und geistlichem Gebiet liegen kann" (CP 102), bezeugen sie es!

\section{IV.}

Im heutigen säkularisierten Kontext werden solche religiösen Kategorien aber inhaltlich näher zu umschreiben sein, so daß ahnbar wird, daß ohne sie etwas verloren geht, weil vielen Zeitgenossen die Zusammenhänge zwischen der hochtechnologisierten Welt der Medien und den Ansprüchen, die von der theologischen Seite her angemeldet werden, nicht ohne weiteres einsichtig sind. Das gilt teilweise bereits für ethische und sozialethische Belange, obwohl diese unmittelbar wahrgenommen werden können. Um so wichtiger ist es, die Medien-Ethik als aktuelle Herausforderung immer wieder einzubringen.

Was die spezifisch theologischen Begründungszusammenhänge anbetrifft, bleibt zu befürchten, daß die in $\mathrm{CP}$ erwähnten Verweise auf Christus als den "vollkomenen Kommunikator" (CP 11) und auf das Geheimnis der heiligen Dreieinigkeit (CP 8) dem meisten nicht viel weiterhelfen. „Werden diese Mysterien nicht trivialisiert, wenn sie so unvermittelt zur Deutung der Massenkommunikation herangezogen werden?", hat schon damals ein kritischer Kommentar gefragt. Nicht notwendigerweise könnte man ihm zur Antwort geben. Etwa mit dem Hinweis, daß Kurt Marti die Lehre von der Dreifaltigkeit neuerdings als „die wohl genialste Leistung der christlichen Theologie" bezeichnet hat, und zwar mit der Begründung, „daß mit ihr die gängigen Vorstellungen von himmlischen Patriarchen, Königen und Autokraten" verabschiedet worden seien. Als Impuls für eine Revitalisierung jener Communio-Ekklesiologie, der sich die Pastoralinstruktion CP in der Folge des 2. Vatikanischen Konzils verpflichtet weiß, ist eine solche Aussage in unserem Zusammenhang durchaus relevant.

Eine derartige theologische und ethische Relevanz für den Medienbereich wird allerdings neu zu begründen sein. Denn sie kann in unseren Verhältnissen nicht mehr vorausgesetzt oder bloß gefordert werden. Schon gar nicht mit Pathos, Beschwörungsformeln und moralisierenden Appellen, denn diese können die erforderliche Fachkompetenz nicht ersetzen. Überzeugendere theologische Ansätze ergeben sich aus der geistig-geistlichen Beschäftigung mit dem Grundbegriff der Kommunikation und einer daraus sich ableitenden 
Kommunikationskultur und Kommunikationstheologie. Dafür gibt es ja tiefe und tragfähige Anhaltspunkte, sowohl in der Dogmatik wie in der Anthropologie. Mindestens wenn man die Ansicht teilt, daß der Mensch nach dem Urbild des dreifaltigen Gottes geschaffen ist und die ganze Menschheit im Sinne des Verständigungswunders von Pfingsten - gegen den Zersplitterungsprozeß von Babel - zur Einheit und damit zur Ausformung eines Einheits- und eines Menschheitsbewußtseins berufen ist. „E possibile incontrarsi a Babele?“, hat der Mailänder Kardinal Carlo Maria Martini in diesem Zusammenhang bei seinem Auftritt am internationalen Medienkongreß von UNDA und OCIC im letzten November gefragt. Damit ist auf eine jener Überlebensfragen hingewiesen, die CP der Aufmerksamkeit empfiehlt, weil sie zu denjenigen gehört, "die die Menschheit im Innersten bewegen" (CP 125).

Die Antwort, die unser Dokument auf diese Frage bereithält, ist ein ungetrübtes, (gut)"gläubiges", idealistisches Ja, das heute kritisch zu durchleuchten ist. Im Prinzip kann zwar kein Zweifel daran bestehen, daß die alten und die neuen Mittel der sozialen Kommunikation zum Entstehen dieses universalen Bewußtseins einen Beitrag leisten können und das teilweise ja auch tun. Die Pastoralinstruktion hat für die Annäherungsversuche der Völker, der Kulturen und der Religionen via Medien nicht nur den Ausdruck „Communio“, sondern auch das Bild vom „runden Tisch“ (CP 19) gebraucht, daß sich als außerordentlich nützlich erwiesen hat. Unabdingbare Voraussetzung dafür ist allerdings, daß nicht die ohnehin finanzstarken Interessengruppen mit ihrem erdrückenden Technologiepotential dieses Gespräch beherrschen, sondern daß auch Minderheiten sich zu Worten melden „dürfen", denn sonst werden die sozialethischen Grundsätze von den ökonomischen zusehends totgeschlagen.

Es gehört zum kirchlichen Medienengagement, sich für diese schwachen und stimmlosen Glieder der Menschheitsfamilie stark zu machen. Warum nicht dadurch, daß ein neuer Versuch unternommen wird, ökumenisch, also zusammen mit anderen gesellschaftlichen und humanitären Kräften, das Projekt einer neuen Weltinformations- und Kommunikationsordnung, das sich um einen fairen Ausgleich zwischen dem Nordenund dem Süden bemüht, wieder auf die Tagesordnung zu setzen? Die WACC (World Association for Christian Communication) scheint bereits solche Initiativen zu unterstützen.

Ähnliche Reflexionsarbeit wäre dem Begriff „Dialog“ und den dialogischen Methoden zu widmen, damit er nicht zu einem bequemen Schlagwort verkommt, das niemanden mehr verpflichtet, nicht einmal zum Zuhören, geschweige denn zur Selbstkritik. „Communio et Progressio" hat dazu bloß den Auftakt gegeben, der weitergezogen werden muß.

Mit diesen inhaltlichen Bezugspunkten und Postulaten bleibt CP mehr als mit der begrenzten Situationswahrnehmung von damals, die heute nicht mehr stimmt - und trotz Auslassungen und Unvollkommenheiten, etwa was ein näheres Eingehen auf die Freiheit des Gewissens betrifft -, ein Dokument des Mutes. Dementsprechend sollte der 20. Geburtstag dazu verleiten, es aus den Regalen hervorzuholen und mit der gegenwärtigen Ausgangslage in der Kommunikationslandschaft, vorab auch in der kirchlichen, zu konfrontieren. Das 
wäre eine dynamische, kritische, keine bloß affirmative, bestätigende, also harmlose Rezeption, die sich mit ein paar Zitaten zufrieden gibt, die häufig bloß eine formale Legitimation erfüllen! Dadurch könnte und müßte die Instruktion sogar zu einem "Dokument des Unmutes" werden, und es hoffentlich so lange bleiben, bis in beiden Bereichen, der Gesellschaft und der Kirche, kommunikations- und menschenfreundlichere Strukturen geschaffen werden. Das kann allerdings noch eine ganze Weile dauern!

\section{Anmerkungen}

1 Vortrag auf der Tagung der Arbeitsgemeinschaft Katholische Presse aus Anlaß des 20jährigen Erscheinens von CP, gehalten am 29.04.1991.

\section{SUMMARY}

The author puts into question whether the statements of „Communio et Progressio" of 1971 are still relevant after the impetous development of communications technology. In his answer he points out that even if some single statements do not appear to be up to date, the general part did not loose its importance. The general declarations of CP concerning the meaning of social communications and the way in which the Church has to live with modern massmedia are still valid.

There is, however, a great deficit in accepting an acting according to these statements especially within the Church itself. Eichenberger refers as an example to the Instruction for theologians of 1990 . Instead of a prevailing mentality of defensive attitudes, an open Investigation of Consciousness and a new describing of our position are required. We have to push $\mathrm{CP}$ again to new life. This would include to work out convincing theological roots in order to make apparent the theological and ethic meanings of masscommunications and to inspire the activities of the Church from these sources. As two examples for fields of action, the author mentions a stronger engagement of the Church for minorities and for a "New World Order of Information and Communication" as it is asked for by the Third World peoples since long.

\section{RÉSUMÉ}

L'auteur demande si les messages de "Communio et Progressio" de 1971 sont encore significatifs aujourd'hui, eu égard à l'evolution fulgurante dans le domaine de la technologie de la communication. Bien que beaucoup de messages, pris séparément, ne paraissent plus d'actualité aujourd'hui, la partie fondamentale n'a toutefois pas perdu de son importance jusqu'à nos jours. Et les déclarations générales de „Communio et Progressio“ relatives à l'importance de la communication sociale et aux rapports qu'il convient à l'Eglise d'entretenir avec les mass media modernes n'ont nullement perdu de leur signification.

Pourtant, c'est justement au sein de l'Eglise elle-même que se révèle aussi un déficit considerable au niveau de la réalisation et de la reception. Eichenberger cite ici à titre d'exemple l'instruction aux théologiens de 1990. Il est donc nécessaire qu'à l'encontre d'une mentalité souvent prédominante dans ses rangs, défensive et bien retranchée sur ses positions, l'Eglise fasse ouvertement le point et son examen de conscience. Il s'agit ainsi, en lui insuffiant de l'efficience, d'aider aujourd'hui à la renaissance de "Communio et Progressio". Pour cela, il est également nécessaire d'élaborer des bases théologiques convaincantes, afin de mieux faire comprendre l'importance théologique et 
éthique de la communication de masse et d'orienter l'action de l'Eglise dans la société pluraliste, en vue p.ex. d'un engagement plus soutenu pour les minorités et pour le "Nouvel ordre mondial de l'information et de la communication", prévu dèjà de longue date par le Tiers-Monde.

\section{RESUMEN}

El autor se pregunta si las afirmaciones de "Communio et Progressio" del año 1971, en vista del rapidísimo desarrollo en el campo de la tecnología de la comunicación, tienen todavía alguna relevancia. Aunque muchas afirmaciones particulares aparecen hoy no adecuadas a nuestro tiempo, las partes sustanciales hasta hoy no han perdido su importancia. Los enunciados generales de „Communio et Progressio“ referentes a la importancia de la comunicación social y al correcto tratamiento de la Iglesia a los medios de masa modernos, no han perdido de ninguna manera su importancia.

Sin embargo se constata un gran deficit en el cumplimiento y en la recepción también dentro de la Iglesia misma. Eichenberger nombra como ejemplo la instrucción a los teólogos del año 1990. Por eso se necesita contra una mentalidad de bunker, a menudo predominante, un análisis de conciencia abierto y con determinandiones de lugar. De lo que se trata pues es de dar nueva vida a "Communio et Progressio" hoy con inyecciones de eficiencia. A ello pertenece la necesidad de elaborar planteamientos teológicos convincentes para hacer claro la relevancia teológia y ética de la comunicación de masas y para orientar la actuación de la Iglesia en esta sociedad pluralista; por ejemplo en referencia a un mayor compromiso por las minorias y por el ${ }_{n}$ nuevo orden de información y comunicación mundial" planteado hace ya tiempo por el Tercero Mundo. 